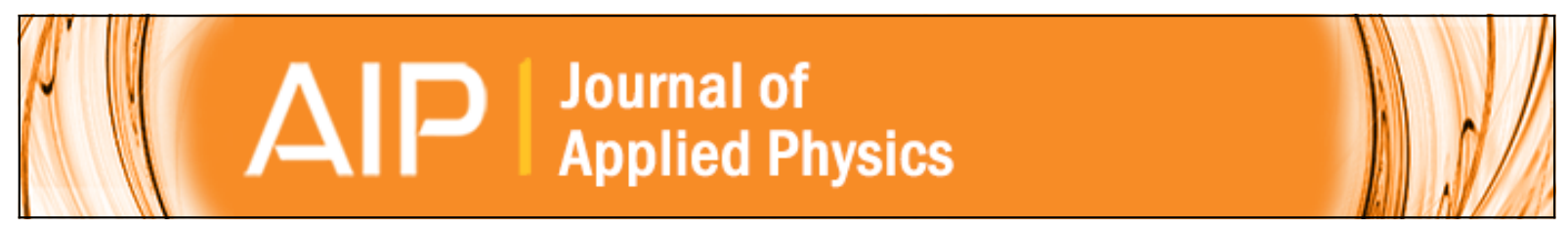

\title{
Optical and microstructural studies of atomically flat ultrathin In-rich In Ga N Ga N multiple quantum wells
}

Soon-Yong Kwon, Hee Jin Kim, Euijoon Yoon, Yudong Jang, Ki-Ju Yee, Donghan Lee, Seoung-Hwan Park, Do-

Young Park, Hyeonsik Cheong, Fabian Rol, and Le Si Dang

Citation: Journal of Applied Physics 103, 063509 (2008); doi: 10.1063/1.2874494

View online: http://dx.doi.org/10.1063/1.2874494

View Table of Contents: http://scitation.aip.org/content/aip/journal/jap/103/6?ver=pdfcov

Published by the AIP Publishing

\section{Articles you may be interested in}

Radiative and nonradiative lifetimes in nonpolar m -plane In x Ga 1 x N Ga N multiple quantum wells grown on GaN templates prepared by lateral epitaxial overgrowth

J. Vac. Sci. Technol. B 25, 1524 (2007); 10.1116/1.2746354

Optical characteristics of a -plane In Ga N Ga N multiple quantum wells with different well widths Appl. Phys. Lett. 90, 181122 (2007); 10.1063/1.2735935

Effect of growth interruption on optical properties of In-rich In Ga N Ga N single quantum well structures J. Appl. Phys. 100, 043520 (2006); 10.1063/1.2220514

Strong potential profile fluctuations and effective localization process in In Ga N Ga N multiple quantum wells grown on $\{101 \mathrm{~m}\}$ faceted surface $\mathrm{GaN}$ template

J. Appl. Phys. 100, 013528 (2006); 10.1063/1.2214211

Efficient radiative recombination and potential profile fluctuations in low-dislocation In Ga N Ga N multiple quantum wells on bulk GaN substrates

J. Appl. Phys. 97, 103507 (2005); 10.1063/1.1897066

A|P| Applied Physics

AIP Letters

is pleased to announce Reuben Collins as its new Editor-in-Chief

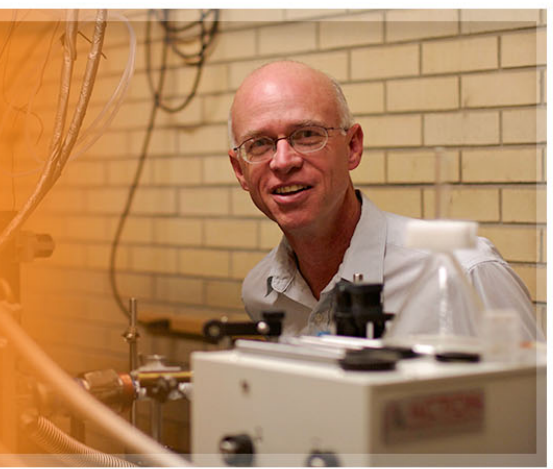




\title{
Optical and microstructural studies of atomically flat ultrathin In-rich InGaN/GaN multiple quantum wells
}

\author{
Soon-Yong Kwon, ${ }^{1, a)}$ Hee Jin Kim, ${ }^{1}$ Euijoon Yoon, ${ }^{1, b)}$ Yudong Jang, ${ }^{2}$ Ki-Ju Yee, ${ }^{2}$ \\ Donghan Lee, ${ }^{2}$ Seoung-Hwan Park, ${ }^{3}$ Do-Young Park, ${ }^{4}$ Hyeonsik Cheong, ${ }^{4}$ Fabian Rol, ${ }^{5}$ \\ and Le Si Dang ${ }^{5}$ \\ ${ }^{1}$ Department of Materials Science and Engineering, Seoul National University, Seoul 151-742, \\ Republic of Korea \\ ${ }^{2}$ Department of Physics, Chungnam National University, Taejon 305-764, Republic of Korea \\ ${ }^{3}$ Department of Electronics Engineering, Catholic University of Daegu, Kyeongsan 712-702, \\ Republic of Korea \\ ${ }_{5}^{4}$ Department of Physics, Sogang University, Seoul 121-742, Republic of Korea \\ ${ }^{5}$ CEA/CNRS/UJF Research Group, Nanophysique et Semiconducteurs, Laboratoire de Spectrométrie \\ Physique (CNRS UMR 5588), Université J. Fourier-Grenoble, 38402 Saint Martin d'Heres Cedex, France
}

(Received 8 September 2007; accepted 18 December 2007; published online 20 March 2008)

\begin{abstract}
Optical and microstructural properties of atomically flat ultrathin In-rich (UTIR) InGaN/GaN multiple quantum well were investigated by means of photoluminescence (PL), time-resolved PL (TRPL), and cathodoluminescence (CL) experiments. The sample exhibits efficient trapping of the photoexcited carriers into quantum wells (QWs) and the effect of internal electric field in the QWs was found negligible by excitation power-dependent PL and TRPL. These phenomena were attributed to the nature of UTIR InGaN QWs, indicating the potential of this system for application in optoelectronic devices. Variation of TRPL lifetime across the PL band and spatially resolved monochromatic CL mapping images strongly suggest that there is micrometer-scale inhomogeneity in effective band gap in UTIR InGaN/GaN QWs, which is originated from two types of localized areas. (C) 2008 American Institute of Physics. [DOI: 10.1063/1.2874494]
\end{abstract}

\section{INTRODUCTION}

Contrary to conventional Ga-rich InGaN alloys, InN and In-rich InGaN alloys are still at the stage of establishing growth techniques and basic physical properties. ${ }^{1}$ It is expected that high defect density would arise from the heterointerface between as-grown $\mathrm{InN}$ and $\mathrm{GaN}$ since the lattice mismatch between them is larger than $10 \%$ and these defects would deteriorate the quality of InN-based heterostructures. ${ }^{1}$ Recently, we have overcome these obstacles and have succeeded in growing high quality and atomically flat ultrathin In-rich (UTIR) InGaN/GaN quantum wells (QWs) with In composition of $60 \%-70 \%$ under quite an elevated growth temperature $\left(730{ }^{\circ} \mathrm{C}\right)$ of $\mathrm{InN} .^{2,3}$ During In-rich InGaN QW growth, only trimethylindium (TMIn) and ammonia $\left(\mathrm{NH}_{3}\right)$ were supplied as precursors; however, strain relaxation and solid-state intermixing occurred, and defective In-rich InGaN QW with thickness fluctuation was formed instead of $\mathrm{InN}$ QW. ${ }^{2,3}$ However, introduction of growth interruption before $\mathrm{GaN}$ capping made the formation of defect-annihilated and atomically flat UTIR InGaN layer possible in QWs because of active decomposition and mass transport process in Inrich $\mathrm{InGaN}$ layer. ${ }^{3}$

UTIR InGaN QWs seem to be significantly free of internal electric field because of ultrathin thickness and/or high residual carrier concentration in the In-rich InGaN well,

\footnotetext{
${ }^{a)}$ Present address: Department of Materials Science and Engineering, University of California at Los Angeles, Los Angeles, CA 90095, USA.

b) Author to whom correspondence should be addressed. Tel.: +82-2-8807169. FAX: +82-2-875-0679. Electronic mail: eyoon@snu.ac.kr.
}

leading to internal electric field effect-free optical property. Also, an efficient carrier trapping into UTIR InGaN QWs is expected because excitons in GaN can be effectively localized at the In-rich InGaN well due to the large band offsets between the well and the barrier and smaller electronegativity of In than $\mathrm{Ga}$, resulting in much stronger oscillator strength of excitons, as in the case of InAs/GaAs system. ${ }^{4}$ Up to now, little knowledge has been acquired about the optical and microstructural properties of InN and/or In-rich InGaN QWs, unlike conventional Ga-rich InGaN QWs. Here, we report a detailed investigation of optical and microstructural properties of UTIR InGaN/GaN multiple QWs (MQWs). An efficient photoexcited carrier trapping into UTIR InGaN QWs was found and the effect of internal electric field in UTIR InGaN QW layer was negligible by excitation power-dependent photoluminescence (PL) and timeresolved PL (TRPL) experiments. Spatially resolved monochromatic cathodoluminescence (CL) mapping images of the MQW and the variation in PL decay time at various energy levels in the PL band indicated the presence of micrometer-scale effective bandgap inhomogeneity.

\section{EXPERIMENTAL}

UTIR InGaN/GaN MQW was grown by metal-organic chemical vapor deposition operating at 300 Torr. The sample was grown on a $c$-plane sapphire substrate. The structure consists of a $2-\mu \mathrm{m}$-thick $\mathrm{GaN}$ buffer layer grown at $1080{ }^{\circ} \mathrm{C}$ and ten period $\mathrm{InGaN}(1 \mathrm{~nm}) / \mathrm{GaN}(20 \mathrm{~nm}) \mathrm{MQW}$ grown at $730{ }^{\circ} \mathrm{C}$. During the growth of UTIR InGaN QW, only TMIn and $\mathrm{NH}_{3}$ were supplied as precursors and $\mathrm{N}_{2}$ carrier gas was 
used. However, strain relaxation as well as solid-state intermixing along growth direction ( $c$-axis) occurred at the early stage of InN deposition on GaN. Medium ion energy scattering (MEIS) measurement showed that the actual In composition in QW layer is 60\%-70\% and the intermixed regions are present at both top and bottom interfaces. ${ }^{5}$ Optical and microstructural properties of the MQW were characterized by PL, TRPL, and CL. PL spectra and decay times of the MQW were obtained at $10 \mathrm{~K}$ at various excitation powers by using a second-harmonic light of a picosecond-pulsed Ti:sapphire laser (fixed wavelength: $367 \mathrm{~nm}$, excitation power: $2 \mathrm{~mW}$, and spot size: $400 \mu \mathrm{m}$ in diameter) and a streak camera with 4 ps resolution. CL experiments were performed using a FEI Quanta 200 scanning electron microscopy equipped with a Jobin Yvon HR460 monochromator and a charge-coupled device camera operating at liquid nitrogen temperature. Typical electron beam voltage and current were $5 \mathrm{kV}$ and $50 \mathrm{pA}$, respectively.

\section{RESULTS AND DISCUSSION}

Figure 1(a) shows the $10 \mathrm{~K}$ PL spectrum of MQW measured by a He-Cd $(325 \mathrm{~nm})$ laser. The near-ultraviolet emission from UTIR InGaN QWs was confirmed by the energy level calculation by Fourier series method using the measured In compositional profile in QWs in our earlier work. ${ }^{5}$ Our recent detailed investigation on electronic structures of UTIR InGaN QWs by eight band $k \cdot p$ method showed that the degree of strain relaxation in our QWs does not significantly change the PL transition energy due to relatively strong confinement and proved the accuracy of In composition obtained by MEIS measurement. ${ }^{6}$ Figure 1(a) shows that there is almost no luminescence in the $\mathrm{GaN}$ band edge position and the integrated PL intensity of GaN band edge is three orders of magnitude smaller than that of QWs. This result strongly indicates an effective bypass of the recombination channels in $\mathrm{GaN}$ and the transfer and capture of photogenerated carriers into QWs would be very fast in this system, which is appropriate for light-emitting applications. Also, the internal quantum efficiency of the current structure was evaluated simply by comparing the ratio of the integrated intensity at low temperature and room temperature and it was $\sim 10 \%$ at the excitation intensity level of $0.1 \mathrm{~W} / \mathrm{cm}^{2}$. This value seems to be low compared to conventional Ga-rich, thicker InGaN QWs; ${ }^{7,8}$ however, internal quantum efficiency is strongly dependent on excitation carrier density ${ }^{7}$ and well/barrier strcuture ${ }^{9}$ especially in $\mathrm{InGaN} / \mathrm{GaN}$ system and further study is currently underway on these issues.

We performed PL and TRPL measurements at $10 \mathrm{~K}$ to evaluate the effect of internal electric field in the MQW at various excitation powers. The PL spectra showed almost no change in peak position $(387 \mathrm{~nm})$ and the normalized line shapes were almost identical when power density was varied from 0.054 to $1.6 \mathrm{~W} / \mathrm{cm}^{2}$, as shown in Fig. 1(b). Furthermore, the TRPL curves showed almost no change as the excitation intensity was varied from 0.054 to $1.6 \mathrm{~W} / \mathrm{cm}^{2}$, as shown in Fig. 1(c). The PL decay times were $1.75 \mathrm{~ns}$, and independent of excitation power and fairly small, compared
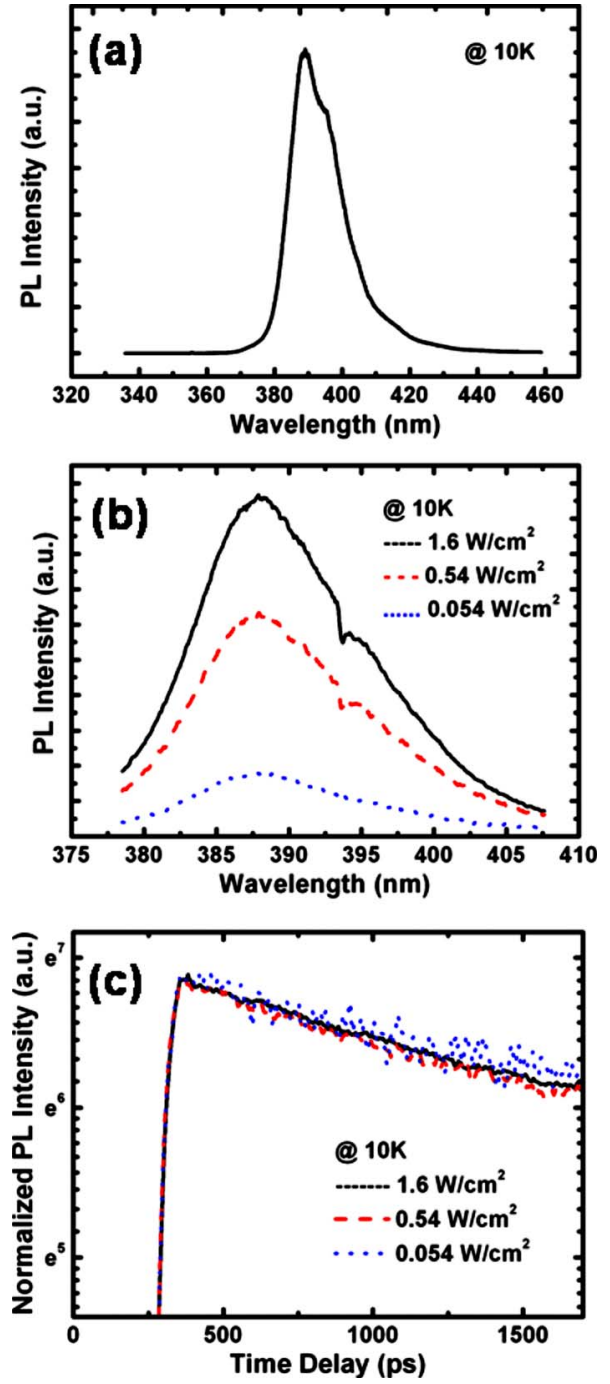

FIG. 1. (Color online) (a) $10 \mathrm{~K}$ PL spectrum, (b) excitation powerdependent $10 \mathrm{~K}$ PL spectra, and (c) $10 \mathrm{~K}$ PL decay curves at $387 \mathrm{~nm}$ from ultrathin In-rich $\mathrm{InGaN} / \mathrm{GaN} \mathrm{MQW}$. The excitation power densities were $0.054,0.54$, and $1.6 \mathrm{~W} / \mathrm{cm}^{2}$ in (b) and (c).

with previous reports on III-nitride heterostructures. ${ }^{10,11}$ In our following study, the electric field effect-free characteristics was also found when we fabricated a light emitting diode structure with UTIR InGaN MQW as an active layer in the current level ranging from 5 to $200 \mathrm{~mA}^{12}$ These results suggest that there was scarcely any internal electric field effect in QWs, despite of the existence of high In content of $60 \%-$ $70 \%$ in the well region. It is clear that internal electric field effect weakened with decrease of well width. ${ }^{13}$ Although the internal electric field in our QWs would be quite weak due to the ultrathin well width, the significant blueshift of $10-15 \mathrm{~nm}$ PL peak is expected with increasing current level to $200 \mathrm{~mA}$ from our calculation of eight-band $k \cdot p$ method. ${ }^{12}$ However, we could not observe any peak shift with increasing current level. It can possibly be attributed to the high residual carrier density over $10^{18} / \mathrm{cm}^{3}$ in In-rich InGaN well, which is enough density to screen the internal electric field. ${ }^{14,15} \mathrm{Up}$ to now, most of reported residual carrier density in $\mathrm{InN}$ is in the order of $10^{18}-10^{21} / \mathrm{cm}^{3}$, which is originated from high density of native defects and donor impurities in 


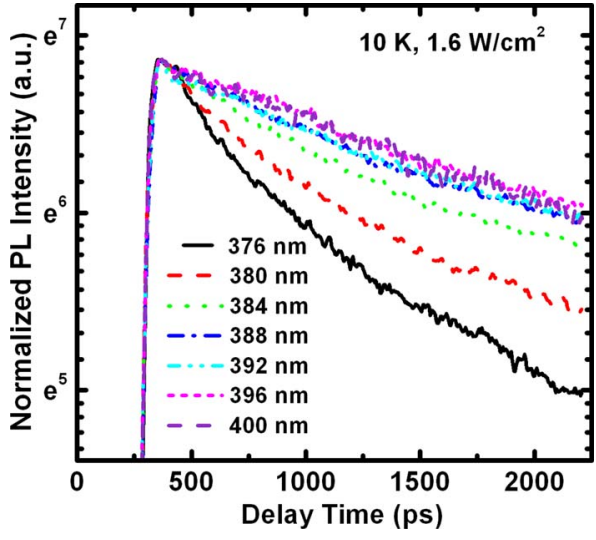

FIG. 2. (Color online) PL decay curves at $10 \mathrm{~K}$ at the various energy positions from ultrathin In-rich InGaN/GaN MQW. The excitation power density was $1.6 \mathrm{~W} / \mathrm{cm}^{2}$.

InN (Ref. 1) and we believe that the residual carrier density in In-rich InGaN well would be also in that high range. Consequently, it is expected that our UTIR InGaN QW is perfectly screened by residual carriers, leading to electric field effect-free optical property.

PL decay times were measured across the PL band to study the carrier dynamics in the MQW at the excitation power density of $1.6 \mathrm{~W} / \mathrm{cm}^{2}$, as shown in Fig. 2. Since the measurement temperature was $10 \mathrm{~K}$, the influence of nonradiative recombination processes could be excluded. ${ }^{16,17}$ Our experimental data were fitted by a stretched exponential line shape $I(t)=I_{1}(0) \exp \left(-t / \tau_{1}\right)+I_{2}(0) \exp \left[\left(-t / \tau_{2}\right)^{\beta}\right]$, which has been used to analyze the emission characteristics of localized system. ${ }^{16,17}$ The parameter $I(t)$ means the PL intensity at time $t, \beta$ is the dimensionality of the localizing centers, and $\tau_{1}$ and $\tau_{2}$ are the initial lifetimes of carriers. Both fast lifetime $\tau_{1}$ and slow lifetime $\tau_{2}$ increased from 0.88 to $1.75 \mathrm{~ns}$ and from 1.60 to $2.49 \mathrm{~ns}$, respectively, as the measurement wavelength was changed from 376 to $400 \mathrm{~nm}$, suggesting carriers trapping at localized states and their transfer from higher energy states to lower energy states. ${ }^{18}$

To confirm the presence of localized states in UTIR InGaN/GaN MQW layer, spatially resolved monochromatic $\mathrm{CL}$ mappings were carried out at $5 \mathrm{~K}$ for the MQW. From the CL images in Fig. 3, we found that the lateral sizes of each bright area were approximately $0.5-2 \mu \mathrm{m}$. The images taken at $376 \mathrm{~nm}$ [Fig. 3(a)] and at $406 \mathrm{~nm}$ [Fig. 3(f)] were quite complementary to each other. As the emission wavelength increased from 376 to $388 \mathrm{~nm}$, the area of bright region gradually increased. At emission wavelengths around $388 \mathrm{~nm}$ [Fig. 3(c)] and $394 \mathrm{~nm}$ [Fig. 3(d)], the entire area was bright, which corresponds to the two peak positions in $10 \mathrm{~K} \mathrm{PL}$, as shown in Fig. 1(a). These results clearly show that there is micrometer-scale inhomogeneity in effective bandgap in UTIR InGaN/GaN MQWs.

Interestingly, the lateral size of localized centers in UTIR InGaN QWs is much larger than that in conventional Garich, thicker InGaN QWs. To our knowledge, the lateral size of localization centers in most of earlier reports about conventional Ga-rich InGaN QWs has been in the order of a few tens of nanometers and it was explained by the result of indium composition undulation. ${ }^{19,20}$ However, in our UTIR
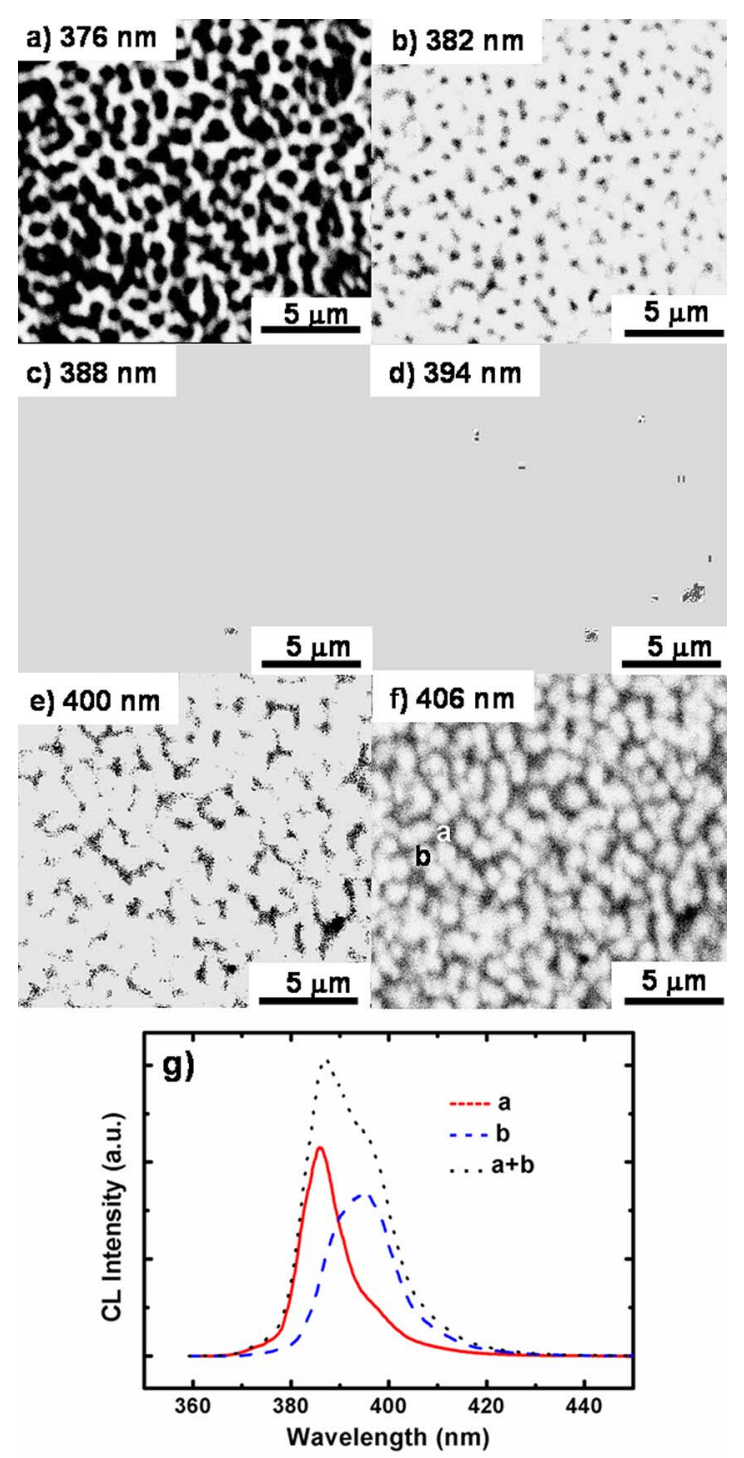

FIG. 3. (Color online) Spatially resolved monochromatic $5 \mathrm{~K}$ CL mapping images taken at (a) $376 \mathrm{~nm}$, (b) $382 \mathrm{~nm}$, (c) $388 \mathrm{~nm}$, (d) $394 \mathrm{~nm}$, (e) $400 \mathrm{~nm}$, and (f) $406 \mathrm{~nm}$. The accelerating voltage was $5 \mathrm{kV}$, indicating luminescence only from MQW region. (g) $5 \mathrm{~K} \mathrm{CL}$ spectra from different areas of the MQW.

InGaN QWs, most of misfit strain in $\mathrm{InN}$ was relieved at the very early stage of InN deposition by threading dislocation formation and also by atomic interdiffusion along growth direction (c-axis). ${ }^{3,5}$ We believe that the formation of quite large localized centers in UTIR InGaN QWs is rather related with monatomic thickness fluctuation in QWs, not with indium composition undulation because general group-III adatom diffusion length at $730{ }^{\circ} \mathrm{C}$ would not be in that high range of micrometer. ${ }^{21}$

In Fig. 3(f) showing $5 \mathrm{~K} \mathrm{CL}$ image taken at $406 \mathrm{~nm}$, we denoted dark and bright areas as (a) and (b), respectively. CL spectra obtained from limited spot areas were shown in Fig. $3(\mathrm{~g})$ and the spot CL peak wavelengths in (a) and (b) were 386 and $394 \mathrm{~nm}$, respectively. The spot CL spectra in other areas were also taken from position to position; however, there were little changes in CL peak position. Also, the widearea integrated CL spectra were almost the same across the whole sample areas. These results can be interpreted as ex- 
istence of two types of localized areas, resulting in effective bandgap inhomogeneity in QWs and the luminescence from each localized areas added to multiple peaks in CL and PL.

\section{CONCLUSION}

In conclusion, the optical and microstructural properties of atomically flat UTIR InGaN/GaN MQW were investigated. We observed an efficient photoexcited carrier trapping into QWs and an electric field effect-free property originated from nature of UTIR InGaN QWs, indicating the potential of this system for light-emitting applications. Micrometer-scale effective bandgap inhomogeneity was present in the QWs and the existence of two types of localized areas was found.

\section{ACKNOWLEDGMENTS}

This work was supported by the Ministry of Science and Technology of Korea through the National Research Laboratory (NRL) Program and National Nano Research and Development Program, by KOSEF through Center for Materials and Processes of Self-Assembly (R11-2005-048-00000-0) and through the Quantum Photonic Science Research Center, by the Ministry of Education through the BK21 Program, and by the Department of the Air Force (AOARD-05-4007).

${ }^{1}$ K. S. A. Butcher and T. L. Tansley, Superlattices Microstruct. 38, 1 (2005).

${ }^{2}$ S.-Y. Kwon, S.-I. Baik, Y.-W. Kim, H. J. Kim, E. Yoon, J.-W. Yoon, H. Cheong, and Y.-S. Park, Appl. Phys. Lett. 86, 192105 (2005).

${ }^{3}$ S.-Y. Kwon, H. J. Kim, H. Na, Y.-W. Kim, H.-C. Seo, H. J. Kim, Y. Shin, E. Yoon, and Y.-S. Park, J. Appl. Phys. 99, 044906 (2006).

${ }^{4}$ M. V. Belousov, N. N. Ledentsov, M. V. Maximov, P. D. Wang, I. N. Yasievich, N. N. Faleev, I. A. Kozin, V. M. Ustinov, P. S. Kop'ev, and C.
M. S. Torres, Phys. Rev. B 51, 14346 (1995).

${ }^{5}$ S.-Y. Kwon, M.-H. Cho, P. Moon, H. J. Kim, H. Na, H.-C. Seo, H. J. Kim, Y. Shin, D. W. Moon, Y. Sun, Y.-H. Cho, and E. Yoon, Phys. Status Solidi A 201, 2818 (2004).

${ }^{6}$ P. Moon, H. J. Kim, S.-Y. Kwon, E. Yoon, S.-H. Park, and J.-P. Leburton, AIP Conf. Proc. 893, 391 (2007).

${ }^{7}$ S. Watanabe, N. Yamada, M. Nagashima, Y. Ueki, C. Sasaki, Y. Yamada, T. Taguchi, K. Tadatomo, H. Okagawa, and H. Kudo, Appl. Phys. Lett. 83, 4906 (2003).

${ }^{8}$ D. M. Graham, P. Dawson, G. R. Chabrol, N. P. Hylton, D. Zhu, M. J. Kappers, C. McAleese, and C. J. Humpreys, J. Appl. Phys. 101, 033516 (2007).

${ }^{9}$ T. Akasaka, H. Gotoh, H. Nakano, and T. Makimoto, Appl. Phys. Lett. 86, 191902 (2005).

${ }^{10}$ G. Pozina, J. P. Bergman, B. Monemar, T. Takeuchi, H. Amano, and I. Akasaki, Phys. Status Solidi A 180, 85 (2000).

${ }^{11}$ P. Hurst, P. Dawson, S. A. Levetas, M. J. Godfrey, I. M. Watson, and G. Duggan, Phys. Status Solidi B 228, 137 (2001).

${ }^{12}$ H. J. Kim, S.-Y. Kwon, P. Moon, S. Choi, Y. S. Jeon, Y. Moon, E. Yoon, S.-H. Park, T. Chung, J. H. Baek, and Y.-S. Park (unpublished).

${ }^{13}$ T. Kuroda and A. Tackeuchi, J. Appl. Phys. 92, 3071 (2002).

${ }^{14}$ S.-H. Park and S.-L. Chuang, Appl. Phys. Lett. 72, 3103 (1998).

${ }^{15}$ J. S. Im, H. Kollmer, O. Gfrorer, J. Off, F. Scholz, and A. Hangleiter, MRS Internet J. Nitride Semicond. Res. 4S1, 20 (1999).

${ }^{16}$ T. Onuma, A. Chakraborty, B. A. Haskell, S. Keller, S. P. DenBaars, J. S. Speck, S. Nakamura, U. K. Mishra, T. Sota, and S. F. Chichibu, Appl. Phys. Lett. 86, 151918 (2005).

${ }^{17}$ T. S. Ko, T. C. Lu, T. C. Wang, M. H. Lo, J. R. Chen, R. C. Gao, H. C. Kuo, S. C. Wang, and J. L. Shen, Appl. Phys. Lett. 90, 181120 (2007).

${ }^{18}$ J. C. Harris, T. Someya, S. Kako, K. Hoshino, and Y. Arakawa, Appl. Phys. Lett. 77, 1005 (2000).

${ }^{19}$ S. Chichibu, K. Wada, and S. Nakamura, Appl. Phys. Lett. 71, 2346 (1997).

${ }^{20}$ D. M. Graham, A. Soltani-Vala, P. Dawson, M. J. Godfrey, T. M. Smeeton, J. S. Barnard, M. J. Kappers, C. J. Humphreys, and E. J. Thrush, J. Appl. Phys. 97, 103508 (2005)

${ }^{21}$ C.-C. Chen, T.-H. Hsueh, Y.-S. Ting, G.-C. Chi, and C.-A. Chang, J. Appl. Phys. 90, 5180 (2001). 